\title{
Molecular-scale shear response of the organic semiconductor $\beta$-DBDCS (100) surface
}

\author{
Rubén Álvarez-Asencio,,${ }^{1,2}$ Jorge S. Moreno-Ramírez, ${ }^{2}$ Carlos Pimentel, ${ }^{3,4}$ Santiago Casado, ${ }^{2}$ Micaela Matta, \\ Johannes Gierschner, ${ }^{2}$ Luca Muccioli, ${ }^{5,6,7}$ Seong-Jun Yoon, ${ }^{8}$ Shinto Varghese, ${ }^{2}$ Soo Young Park, ${ }^{8}$ \\ Enrico Gnecco, ${ }^{9, *}$ and Carlos M. Pina ${ }^{3,4}$ \\ ${ }^{1}$ SP Technical Research Institute of Sweden, SP Chemistry, Materials and Surfaces, SE-114 86 Stockholm, Sweden \\ ${ }^{2}$ IMDEA Nanoscience, Campus Universitario de Cantoblanco, Calle Faraday 9, E-28049 Madrid, Spain \\ ${ }^{3}$ Departamento de Cristalografía y Mineralogía, Facultad de Ciencias Geologícas, Universidad Complutense de Madrid, \\ Calle José Antonio Novais, 2, E-28040 Madrid, Spain \\ ${ }^{4}$ Instituto de Geociencias IGEO (UCM-CSIC), Calle José Antonio Novais, 2, E-28040 Madrid, Spain \\ ${ }^{5}$ Laboratoire de Chimie des Polymères Organiques, UMR 5629, Université de Bordeaux, Pessac, France \\ ${ }^{6}$ Institut des Sciences Moléculaires, UMR 5255, Université de Bordeaux, Talence, France \\ ${ }^{7}$ Department of Industrial Chemistry “Toso Montanari," Università di Bologna, I-40136 Bologna, Italy \\ ${ }^{8}$ Center for Supramolecular Optoelectronic Materials, Department of Materials Science and Engineering, \\ Seoul National University, ENG 445, Seoul 151-744, Korea \\ ${ }^{9}$ Otto Schott Institute of Materials Research, Friedrich Schiller University Jena, D-07743 Jena, Germany
}

(Received 2 March 2017; revised manuscript received 24 August 2017; published 13 September 2017)

\begin{abstract}
In this work we present friction-force microscopy (FFM) lattice-resolved images acquired on the (100) facet of the semiconductor organic oligomer $\left(2 Z, 2^{\prime} Z\right)-3,3^{\prime}$-(1,4-phenylene)bis(2-(4-butoxyphenyl)acrylonitrile) $(\beta$ DBDCS) crystal in water at room temperature. Stick-slip contrast, lateral contact stiffness, and friction forces are found to depend strongly on the sliding direction due to the anisotropic packing of the molecular chains forming the crystal surface along the [010] and [001] directions. The anisotropy also causes the maximum value of the normal force applicable before wearing to increase by a factor of 3 when the scan is performed along the [001] direction on the (100) face. Altogether, our results contribute to achieving a better understanding of the molecular origin of friction anisotropy on soft crystalline surfaces, which has been often hypothesized but rarely investigated in the literature.
\end{abstract}

DOI: 10.1103/PhysRevB.96.115422

\section{INTRODUCTION}

Friction anisotropy has been a research focus since the 1960s, when Steijn [1] and Bowden et al. [2] independently published their pioneering work on ionic crystals. The contrasting results reported by the two groups could be related to the variation in the wear rates with the sliding directions, as done by Sawyer et al. using more accurate instrumentation [3]. Friction anisotropy is also observed in the absence of abrasive wear. This was demonstrated by Hirano et al. [4] for the friction dependence on the crystallographic orientation on a mica surface, as observed by friction-force microscopy (FFM). Since then, a great deal of effort has been deployed to fully understand this effect. Friction anisotropy has been studied extensively using FFM on several systems, e.g., graphite [5], lipid monolayers [6-8], fullerene islands [9], polytetrafluoroethylene (PTFE) [10], graphene [11,12], $\beta$-alanine [13], and even quasicrystal surfaces [14]. Friction anisotropy has been related to molecular organizations, topological properties, and deformation processes such as the orientation of the constituent alkyl chains [6] and the aperiodicity along specific directions in the case of quasicrystal surfaces [14]. In the case of graphene a "puckering" effect was suggested by Choi et al. [11] but was later dismissed by Gallagher et al., who rather related the friction anisotropy to the presence of adsorbates on the surface [12]. On graphite [15,16] the anisotropy can be simply associated with the crystalline structure of the substrate.

\footnotetext{
*enrico.gnecco@uni-jena.de
}

It is also worth mentioning the combined molecular dynamic (MD) simulations and microtribological measurements at interfaces of PTFE films by Jang et al. [10]. In that work, the molecular structural orientation of the PTFE chains was found to strongly affect tribological properties. When the chains are sheared parallel to their backbones, friction forces decrease, and interfacial slip is enhanced. In contrast, when the shear direction is perpendicular to the backbones, friction and wear increase due to molecular reorientation. MD simulations have been also reported on quasicrystal surfaces by Ye et al., who, interestingly, could recover friction anisotropy only after passivating the probing tip with chains of hexadecane thiol [17].

Lattice-resolved friction maps are very important for supporting any theoretical interpretations of the mechanisms leading to friction anisotropy. However, lattice resolution is difficult to achieve in ambient conditions due to the formation of a water meniscus between the probing tip and surface because of capillary condensation. A costly alternative is to measure the friction in ultrahigh-vacuum conditions [12,18], but as shown by some of us [19-22], this obstacle can also be overcome if the tip is completely immersed in a liquid (e.g., water), so that capillary bridges between the tip and surface are not formed. In this way, we were able to relate the strong friction anisotropy observed on the (104) cleavage surface of calcite and dolomite to the pathways followed by the tip driven along different scan directions [21]. This work led to an understanding of the relation between surface commensurability and friction anisotropy with submolecular resolution of a kyanite 
surface [23]. We were also able to perform structural characterization on soft materials, such as organic semiconductor crystal surfaces [20]. In the present work, we have extended this kind of analysis by focusing on the mechanical anisotropy of the organic semiconductor $\left(2 Z, 2^{\prime} Z\right)-3,3^{\prime}$-(1,4-phenylene)bis(2(4-butoxyphenyl)acrylonitrile) ( $\beta$-DBDCS) crystal. Organic semiconductors have found much interest as alternatives to their inorganic counterparts as easily processable materials with tunable electronic properties, with applications in cheap, thin, light-weight, and flexible (opto)electronics such as fieldeffect transistors, light-emitting diodes, solar cells, and lasing, sensing, or optical memory devices [24-26]. Although the (opto)electronic properties of conjugated materials were studied for a multitude of compounds, the mechanical response of these semiconductors, especially at the nanoscale, has attracted only limited attention [27-33], even though important correlations may exist. A striking example is mechanochromism [34-37], i.e., piezo-induced color change through crystal-tocrystal or crystal-to-amorphous transitions, which are often reversed through other external stimuli (vapor, temperature). A detailed understanding of piezo-induced phase transitions requires insight into crystal formation controlled by nanomechanical properties, which can be studied by atomic-force microscopy (AFM) at the nanoscale [27]. Furthermore, the formation of grain boundaries, which control the microtexture and thus, to a large extent, charge and exciton transport in the devices [38,39], is directly related to the nanomechanical properties at the surface of the crystallites, where AFM provides again nanoscale structural information [28,30]. This equally holds for thin films and crystal surfaces [19,20], where, for instance, the quality of lasing in the crystal is controlled by habit (relative shape) and tracht (size, shape, and homogeneity of the facets) of the crystal as well as by the orientation of the molecules with respect to the crystal facets $[40,41]$. All these parameters are controlled by the molecular electronic and steric demands. Therefore, a fundamental understanding of nanomechanical properties is crucial for materials design. With this in mind, we have studied the response of $\beta$-DBDCS to the shear stress exerted by the AFM tip. As in previously reported cases [19,21,22], measurements are performed in water, where the enhanced lateral resolution allows us to draw quantitative conclusions and precisely relate friction coefficients and lateral contact stiffness to the scan direction. To support the experimental results we have also performed free-energy calculations, the details of which are provided below.

\section{MATERIALS AND METHODS}

\section{A. $\beta$-DBDCS samples}

The present study focuses on monocrystalline samples. The synthesis, chemical characterization, details on single-crystal growth, and $\mathrm{x}$-ray analysis of $\beta$-DBDCS were described elsewhere [29]. Single crystals were grown from a solvent mixture of ethanol and ethyl acetate $(1: 1)$ by slow evaporation. The monoclinic $(P 21 / c)$ crystals have the following lattice parameters: $a=2.78 \mathrm{~nm}, b=0.70 \mathrm{~nm}, c=0.66 \mathrm{~nm}, \alpha=$ $\gamma=90.0^{\circ}$, and $\beta=90.1^{\circ}$. The crystallographic data were obtained from the Cambridge Crystallographic Data Centre
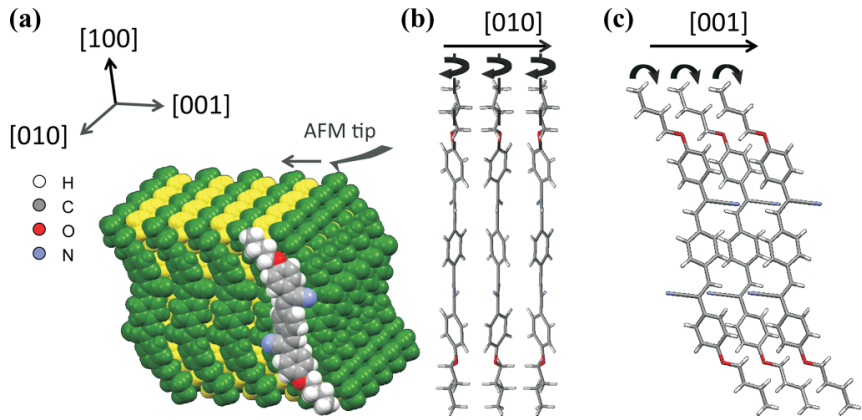

FIG. 1. Crystal structure of the $\beta$-DBDCS molecules according to SC-XRD analysis. (a) Oblique top view of the surface, i.e., (100) plane (space-fill model), (b) side view along the [010] direction, and (c) side view along the [001] direction (capped stick models). Black curved arrows illustrate the rotation [in (b)] and bending [in (c)] of the $\mathrm{C}-\mathrm{C}$ chain ends due to the interaction with the probing tip.

(CCDC: 969314, 969315). Combined AFM, single-crystal (SC), and specular x-ray diffraction (XRD) analysis showed that the $\beta$-DBDCS large crystal face studied in the current work corresponds to the (100) plane [40]. The crystals are expected to be cleavable on the (100) plane because the surface is formed by floppy alkyl chains [see Fig. 1(a)]. Within the $\beta$-DBDCS (100) plane the alkyl chains are not densely packed, so there is some flexibility to move the chains in both the [010] and [001] directions, as presented in Figs. 1(b) and 1(c), respectively.

\section{B. Atomic-force microscopy}

The (100) faces of $\beta$-DBDCS crystals were scanned at room temperature with two commercial AFMs (Nanoscope Multimode IIIa, Veeco Instruments, Santa Barbara, California, and Nanowizard II, JPK Instruments, Berlin, Germany). A monocrystal surface was first characterized by contact mode in air conditions using OMCL-RC800PSA probes (resonance frequency of $18 \mathrm{kHz}$, force constant of $0.05 \mathrm{~N} / \mathrm{m}$ ) from Olympus (Tokyo, Japan). The imaging was performed with a scan rate of $0.5 \mathrm{~Hz}$, a scan size of $6 \times 4 \mu \mathrm{m}^{2}$, and a grid of $512 \times 343$ pixels $^{2}$. To achieve lattice resolution the sample was placed in a commercially available AFM liquid cell which was subsequently filled with deionized water (Milli-Q Millipore, Millipore, Molsheim, France, with specific resistivity of $18 \mathrm{M} \Omega \mathrm{cm}$ ) and scanned in contact mode. In this case, $\mathrm{V}$-shaped silicon nitride cantilevers with integrated ultrasharp silicon tips SNL-10 D (nominal resonance frequency of $18 \mathrm{kHz}$, nominal force constant of $0.06 \mathrm{~N} / \mathrm{m}$, tip radius of $10 \mathrm{~nm}$ ) from Bruker (Camarillo, California) were used. FFM images were obtained with the fast scan direction oriented along either the [010] direction or the [001] direction, with different load values up to $7 \mathrm{nN}$, a scan rate of $61 \mathrm{~Hz}$, a scan size of $10 \times 10 \mathrm{~nm}^{2}$, and a resolution of $512 \times 512$ pixels. All the images were processed using NANOTEC WSXM V4.0 BETA 8.1 (WSxM Solutions, Madrid, Spain) [42], JPK DATA PROCESSING V1.2 (JPK Instruments USA, Inc., Carpintera, California), and NANOSCOPE ANALYSIS V1.50 (Bruker, Santa Barbara, California). The torsion of the cantilever spring holding the probing tip during forward and backward scanning was converted into friction force, and the 
deflection of the cantilever was converted into normal force as described elsewhere [43]. The torsional constant of the cantilever was estimated as in Ref. [44].

\section{Free-energy calculations}

All free-energy calculations were performed with the NAMD code [45]. The $\beta$-DBDCS molecule was modeled with the generalized AMBER force field [46] completed with B3LYP/6-311G* atomic point charges. Torsional potentials for the phenyl-vinyl and phenyl- $\mathrm{CC} \equiv \mathrm{N}$ rotation and for all the dihedrals along the oxybutyl chain were calculated with relaxed scans at the B3LYP/6-311G* level and inserted in the force field (see Ref. [47] for the details of the procedure). Standard Lorentz-Berthelot mixing rules [contact distances $\sigma_{i j}=\left(\sigma_{i}+\sigma_{j}\right) / 2$, well widths $\left.\epsilon_{i j}=\sqrt{\epsilon_{i} \epsilon_{j}}\right]$ were employed for modeling Lennard-Jones (LJ) interaction between different atom types (e.g., for tip-surface interaction). A radial cutoff of $1.6 \mathrm{~nm}$ was used for LJ interactions, and long-range electrostatic interactions were calculated with the smoothparticle-mesh Ewald method as implemented in NAMD [45].

A monoclinic supercell made of 256 molecules $(2 \times 8 \times 8$ unit cells) was equilibrated for $0.2 \mathrm{~ns}$ in the $N V T$ ensemble at $T=173 \mathrm{~K}$ and then for $1 \mathrm{~ns}$ in the $N P T$ ensemble at $p=1$ atm, with three-dimensional periodic boundary conditions. The simulated cell parameters $(a=2.783 \mathrm{~nm}, b=$ $0.692 \mathrm{~nm}, c=6.89 \AA, \alpha=90^{\circ}, \beta=90.07^{\circ}, \gamma=90^{\circ}$, with standard deviations below $0.01 \%$ ) showed good agreement with experimental values measured at the same temperature (reported in Sec. II A). The system was then reequilibrated at $300 \mathrm{~K}$ for $10 \mathrm{~ns}$. Finally, the molecules belonging to the lower monolayer were frozen, while the upper monolayer was relaxed for a further $5 \mathrm{~ns}$, with periodic boundary conditions on the monolayer plane and vacuum on top of it.

Adaptive biasing force $(\mathrm{ABF})$ simulations were then run for $10 \mathrm{~ns}$ to explore (i) the energetic corrugation of the surface and (ii) the potential energy required for displacing an alkyl chain exposed to the surface, in both cases under constant volume and temperature conditions ( $T=300 \mathrm{~K}$ ). During ABF simulations the calculated mean forces, acting on user-defined collective variables, are compensated by an opposite and equal force at each (discretized) value of the variables [48]. This stratagem helps the system explore the entire phase space and overcome high potential-energy barriers. The free-energy surface is then obtained by integrating the measured force along the collective variables' (hyper)surface. The corrugation of the surface was probed with a spherical Lennard-Jones probe with an equilibrium radius $R_{e q}=0.3 \mathrm{~nm}(\sigma=0.5345 \mathrm{~nm})$ and $\epsilon=0.2 \mathrm{kcal} / \mathrm{mol}$. The collective variables explored were the components of the distance vector from joining the sphere to a specific methyl terminal group exposed to the surface. The free energy (or potential of the mean force) experienced by the tip sampled within a volume of about $0.7 \times 0.7 \times 2.2 \mathrm{~nm}^{3}$ along the [001], [010], and [001] axes was averaged on the (001) plane at each value of the distance from the surface in order to locate the position of maximum tip-surface interaction and identify the repulsive region of the potential. Figure 2(c) shows a full $x, y$ map in such a region, corresponding to the spherical tip being moved $0.05 \mathrm{~nm}$ closer to the surface with respect to the position of maximum interaction. In a further simulation
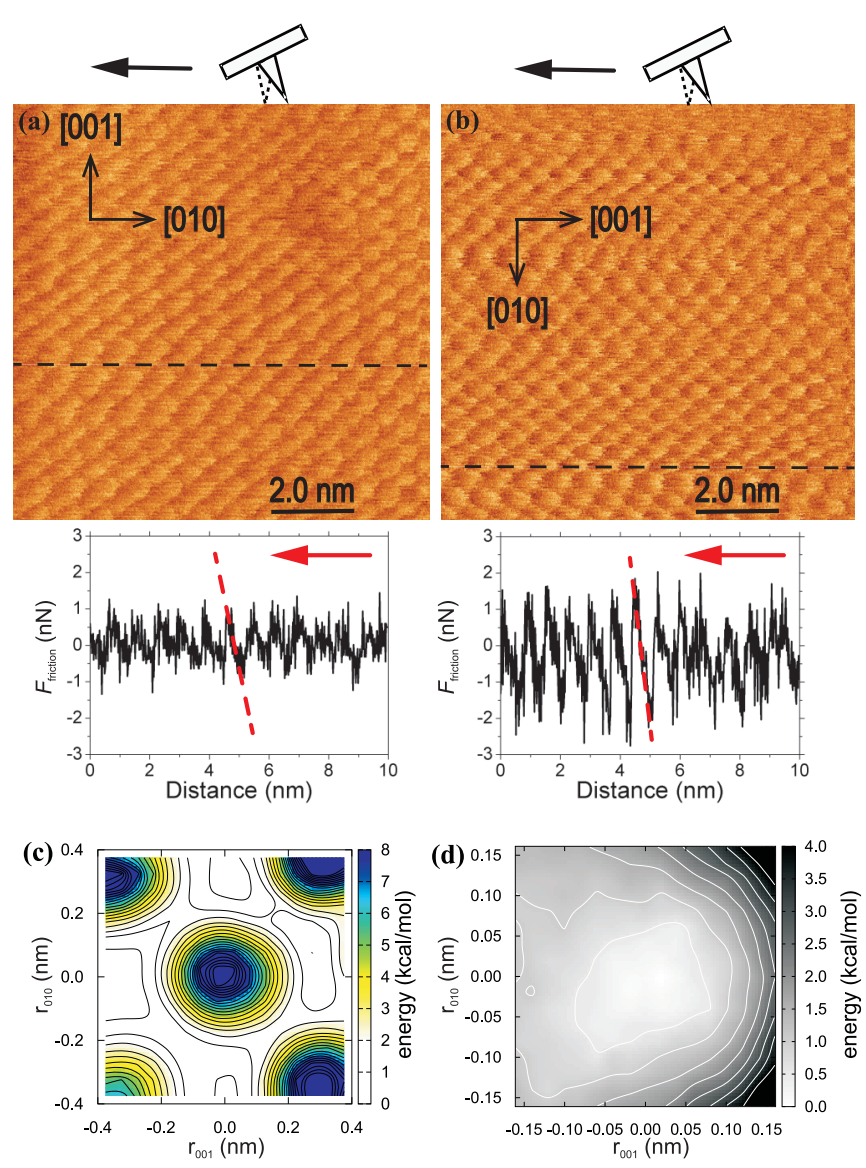

FIG. 2. Friction-force HR images of a $10 \times 10 \mathrm{~nm}^{2} \beta$-DBDCS single crystal scanned along (a) the [010] and (b) [001] directions. These images were constructed from retrace friction signals while applying a load force of around $1.2 \mathrm{nN}$. The profiles below show the variation of the friction force through cross sections of $10 \mathrm{~nm}$ (dashed black line). The red arrow represents the tip scan direction, and the dashed red line shows a stick event. (c) Calculated freeenergy map of a surface unit cell probed with a Lennard-Jones sphere. (d) Free-energy map for displacing a $\beta$-DBDCS terminal methyl group along the [010] and [001] directions. The equilibrium position is located at the center of the map.

experiment, we measured instead the free energy required for displacing an alkyl chain, exposed at the surface, away from its equilibrium position. In this case the collective variables consist of the $x, y$ distance of a methyl group exposed to the surface, with the minimum-energy position set at $(0,0)$. Hence, in Fig. 2(d) each point on the map represents the position of the methyl with respect to the one at rest, with the color giving the corresponding free energy.

\section{RESULTS AND DISCUSSION}

As a first step, we investigated the surface of a single-crystal $\beta$-DBDCS by contact-mode AFM in air. As seen in Fig. 3(a), the surface is formed by atomically flat terraces with a width of a few micrometers. A characteristic terrace height of around $2.8 \mathrm{~nm}$ has been measured [Fig. 3(b)], which coincides with the length of lattice constant $a=2.82 \mathrm{~nm}$ [40]. 
(a)

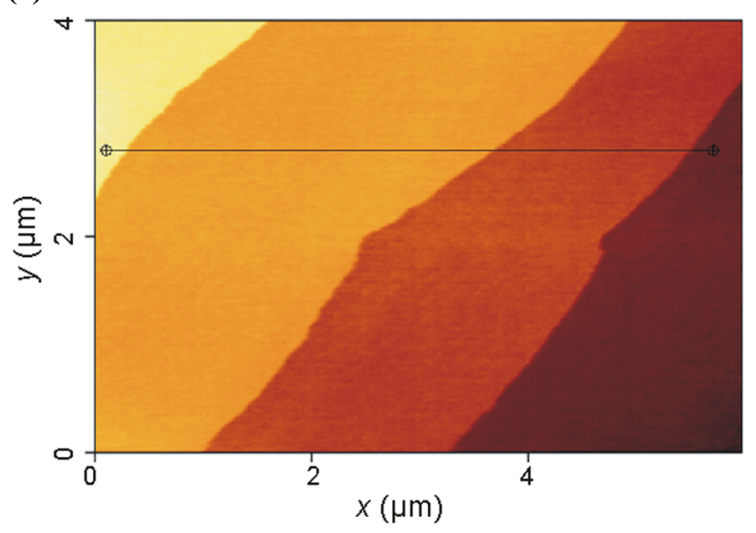

(b)

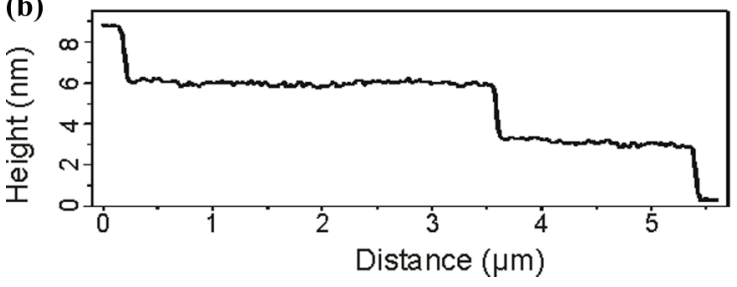

FIG. 3. (a) AFM topography of a $\beta$-DBDCS single-crystal surface. (b) Cross section along the black line in (a) showing the height of the surface terraces.

Figure 2 shows two representative $10 \times 10 \mathrm{~nm}^{2}$ friction force images of the $\beta$-DBDCS surface measured along the [010] and [001] directions in water. The sawtooth profiles of the friction signal are caused by the stick-slip motion [49] of the AFM tip. While the tip scans the sample surface, the cantilever twists periodically. This torsion is determined by the lateral contact stiffness $k_{\text {lat }}$ and the tip-sample interaction potential $U_{0}$. When the angle of torsion reaches a critical value, the tip jumps into a new pinning site on the crystal lattice [50]. By detecting and processing this periodic torsion, friction maps can be generated as shown in Fig. 2. Rectangular unit cells are clearly defined by the average repetition distance of the stick-slip motion $\lambda$ with values of $0.77 \pm 0.02 \mathrm{~nm}$ along [010] [Fig. 2(a)] and $0.71 \pm 0.02 \mathrm{~nm}$ along [001] [Fig. 2(b)]. These values are compatible with those of lattice constants $b$ and $c$ of the $\beta$-DBDCS crystal, so that the pinning sites can be reasonably associated with the periodicity of the terminal methyl groups in Fig. 1.

Even though the same normal force $(1.2 \mathrm{nN})$ was applied while scanning along the [010] and [001] directions, the stick-slip profiles appear to be different. The profile is closer to a sinusoidal curve in the former case, while it resembles a sawtooth shape in the latter. The static friction value $F_{\max }$, as calculated from the average of the lateral force peaks, reaches values of $0.51 \pm 0.17$ and $1.14 \pm 0.38 \mathrm{nN}$ for the directions [010] and [001], respectively. From the average values of the slopes of the friction forces vs distances (profiles in Fig. 2), we also calculated the effective lateral stiffness $k_{\text {exp }}$, with values of $1.41 \pm 0.40 \mathrm{~N} / \mathrm{m}$ for the [010] direction and $3.56 \pm 0.79 \mathrm{~N} / \mathrm{m}$ for the [001] direction. As discussed in Ref. [51], this quantity represents a series of springs corresponding to the lateral deflection of the cantilever, of the tip apex, and, very important
TABLE I. Set of frictional and mechanical coefficients obtained from the sawtooth profiles along the directions [010] and [001] in Fig. 2.

\begin{tabular}{lcccccc}
\hline \hline Direction & $\lambda(\mathrm{nm})$ & $F_{\max }(\mathrm{nN})$ & $k_{\exp }(\mathrm{N} / \mathrm{m})$ & $\eta$ & $U_{0}(\mathrm{eV})$ & $k_{\text {lat }}(\mathrm{N} / \mathrm{m})$ \\
\hline$[010]$ & 0.77 & 0.51 & 1.41 & 1.97 & 0.79 & 2.13 \\
{$[001]$} & 0.71 & 1.14 & 3.56 & 1.76 & 1.61 & 5.74 \\
\hline \hline
\end{tabular}

in the present case, of the sample region laterally sheared by the tip. From $k_{\text {exp }}$, a characteristic friction parameter $\eta$ can be calculated according to the relation [52]

$$
\eta=\frac{2 \pi F_{\max }}{k_{\exp } \lambda}-1 .
$$

Here, the values $\eta=1.97$ and $\eta=1.76$ can be associated with the directions [010] and [001], respectively. The parameter $\eta$ quantifies the ratio between the corrugation of the tip-sample interaction potential $U_{0}$ and the elastic energy stored in the deformed contact. The values of $U_{0}$ are easily calculated as

$$
U_{0}=\frac{\lambda F_{\max }}{\pi},
$$

resulting in $1.26 \times 10^{-19} \mathrm{~J}=0.79 \mathrm{eV}$ and $2.58 \times 10^{-19}$ $\mathrm{J}=1.61 \mathrm{eV}$ for the directions [010] and [001], respectively. Finally, once the value of $\eta$ is known, the lateral contact stiffness can be determined by

$$
k_{\text {lat }}=\left(1+\frac{1}{\eta}\right) k_{\exp }
$$

Whereas on hard surfaces the contribution of the second term in parentheses is usually negligible, that is not the case on $\beta$-DBDCS. Indeed, Eq. (3) yields values of $k_{\text {lat }}$ suggesting that the surface is almost three times softer along the [010] direction $\left(k_{\text {lat }}=2.13 \mathrm{~N} / \mathrm{m}\right)$ than along the [001] direction $\left(k_{\text {lat }}=5.74 \mathrm{~N} / \mathrm{m}\right)$. The results are summarized in Table I.

The pronounced anisotropy along [010] vs [001] might be surprising at a first glance since the lattice constants of the crystal in the $b$ and $c$ directions are very similar (see above). Also the calculated free-energy maps of the surface, obtained with ABF simulations using a spherical Lennard-Jones probe of $\sigma=0.5345 \mathrm{~nm}$ and $\epsilon=0.2 \mathrm{kcal} / \mathrm{mol}$, do not exhibit anisotropy. In Fig. 2(c) we show an example of such maps, corresponding to a vertical position of the probe $0.05 \mathrm{~nm}$ inside the repulsive region of the potential: the four rather symmetric peaks correspond to the position of the oxybutyl chains, which we identify as the source of corrugation of the energy surface. However, the corrugation along the horizontal [001] direction is, in the limit of the resolution of the calculation, identical to the one along the vertical [010] direction.

Rather, the origin of the anisotropy should be sought in the orientation of the alkyl chains, which is determined by the arrangement of the molecular backbones, mainly directed by dipolar interactions of the functional cyano groups [53]. This brings the conjugated backbones of the molecules into a tilted and slipped $\beta$-stacked arrangement along $c$. The plane formed by the backbone of the alkyl chains essentially coincides with the (010) plane [compare Figs. 1(a), 1(b), and 1(c)], and the alkyl chains enclose an angle of about $20^{\circ}$ with the $a$ 
(a)
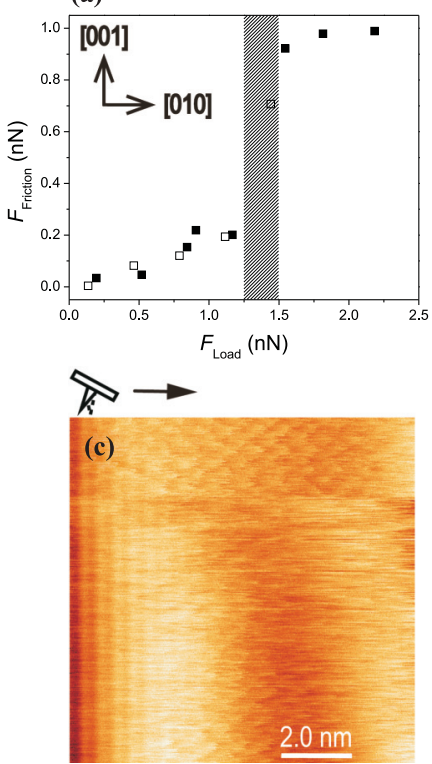

FIG. 4. Dynamic friction force as a function of the applied load while scanning along the (a) [010] and (b) [001] directions. The points correspond to results from two series of independent data sets (represented by different symbols). Friction-force images corresponding to normal forces of (c) 2.5 and (d) $6.8 \mathrm{nN}$, i.e., slightly above the transition region in (a) and inside the corresponding region in (b). Note the sudden change in contrast, which, in line with Ref. [54], we attribute to thermally activated onset of surface wear.

axis [Fig. 1(c)]. Regarding the deformation mechanism of the $\beta$-DBDCS surface, a comparison of Figs. 1(b) and 1(c) suggests that a shear force in [010] produces a rotation of C-C (covalent) bonds, mostly on the chain terminus head. In contrast, along the close-packed [001] direction, the shear force promotes the bending of these C-C (covalent) bonds, similar to a bristle in a hairbrush. The promotion of rotation along the [001] direction is prevented by the tilt of the internal structure of the chain presented in Fig. 1(c). As a consequence, the energy barriers to overcome and the stiffness observed in the stick phase are expected to be larger when the tip is driven along the [001] direction, as observed in our measurements. In order to confirm this mechanism, we also calculated the free-energy map for displacing one of the surface terminal alkyls from its equilibrium position, as shown in Fig. 2(d). More specifically, the coordinates on the map represent the position of the terminal methyl group with respect to the equilibrium one at the origin, and the color gives the relative free-energy difference. Unlike in Fig. 2(c), here, the anisotropy is apparent from the different curvatures of the energy surface along [010] and [001], with curvature being steepest for [001] displacements, in accord with the higher friction measured along the latter direction.

While scanning along the [010] and [001] directions of the single $\beta$-DBDCS crystal, we also recognized an anisotropic response of the dynamic friction force $F_{\mathrm{dyn}}$ as a function of the normal load. Note that $F_{\mathrm{dyn}}$ corresponds to the energy dissipation divided by the distance moved and is simply equal to the average value of the friction curves.

Figure 4 shows a summary of these results. In Fig. 4(a) a linear relationship between the dynamic friction force along [010] and the normal force is observed at low loads, yielding a friction coefficient $\left(\mu=F_{\text {dyn }} / F_{\text {load }}\right)$ of $0.12 \pm 0.04$ (calculated from the slope of the $F_{\text {dyn }}$ vs $F_{\text {load }}$ curve). After overcoming a normal force threshold of $1.25 \mathrm{nN}$, friction suddenly increases until about $1.6 \mathrm{nN}$. Figure 4(b) shows a qualitatively similar response when scanning along the [001] direction, although in this case the increment in friction occurs at a much higher normal force (about $6 \mathrm{nN}$ ). It is also less pronounced, whereas the value of the friction coefficient at low loads (below $2.5 \mathrm{nN}) \mu=0.08 \pm 0.04$ is comparable. When the normal force overcomes the threshold values of 1.25 and $6 \mathrm{nN}$ in the [010] and [001] directions, respectively, the sudden increase in friction suggests that the $\beta$-DBDCS surface microstructure can no longer hold the shear stress applied by the tip, and the surface becomes irreversibly deformed. Note that the original resolution was always recovered after reducing the loading force to the initial values, attesting that the tip apex was not modified by the repeated loading and unloading cycles.

The onset of surface damage is confirmed by Figs. 4(c) and 4(d). Here, two friction-force images were taken along the [010] direction with a normal force of around $2.5 \mathrm{nN}$ and along the [001] direction with a normal force of around $6.8 \mathrm{nN}$. After a few scan lines, a curved offset [Figs. 4(c) and 4(d) and their corresponding profiles] suddenly added to the regular stick-slip profile. Interestingly, a similar effect was observed on alkali halide ( $\mathrm{KBr}$ ) surfaces in ultrahigh vacuum [54], related to the irreversible change of the surface. In Ref. [54] the $\mathrm{K}^{+}$and $\mathrm{Br}^{-}$ions removed by the tip were indeed found to grow homoepitaxially beside the worn off areas, suggesting an atom-by-atom attrition process (see also Ref. [55]). The chain structure of $\beta$-DBDCS makes the scenario much more complex in the present case. In this context, it is interesting to observe that, in their microtribological measurements, Jang et al. [10] found an almost instantaneous wear onset when two surfaces formed by PTFE chains were set into motion perpendicular to the chains direction, whereas the two surfaces could sustain 150 cycles of sliding without damage when the scan direction was rotated by $90^{\circ}$ parallel to the chains. At the same time, the accompanying MD simulations suggested, after $40 \mathrm{~nm}$ of sliding, a consistent displacement of $\sim 6 \mathrm{~nm}$ of the uppermost row of carbon atoms in the chains in the former case but not in the latter one.

In our system, the chains are not parallel to the free surface of the $\beta$-DBDCS crystal, and a direct comparison with Ref. [10] is not possible. Nevertheless, our lattice-resolved images provide additional information on the lateral stiffness anisotropy, where the high flexibility (and low stiffness) of the molecular chains along the [010] direction can be assumed to be related to the preferential deformation of the crystal surface along this direction.

\section{CONCLUSIONS}

Lattice-resolved FFM in water was performed on surfaces of $\beta$-DBDCS crystals in order to understand the influence of the surface microstructure on the nanomechanical response of the crystal surface when sheared along different directions. A stick-slip behavior was observed, with a periodicity dictated by the crystal lattice parameters of $\beta$-DBDCS. In the experiment, 
the static friction force $F_{\max }$ and the lateral contact stiffness $k_{\text {lat }}$ are lower (see Table I) along the [010] direction than along [001], which corresponds to a lower shear resistance of the crystal surface along the former direction. The inspection of the crystal cell suggests that when sliding along [001], a closer packing of the alkyl chains and consequent "steric constriction" should be encountered. The anisotropic response is further confirmed experimentally by the evolution of dynamic friction forces vs normal forces in the two directions. In this case, clearly different normal-force thresholds for surface damage appear (1.25 and $6 \mathrm{nN}$, respectively), which are also consistent with the arrangement and bendability of the alkyl chains forming the crystal surface. Among the possible anisotropy mechanisms mentioned in the Introduction, the closest to our results is the shear-induced structural orientation discussed in Ref. [10]. Note that in our measurements, lattice resolution could be achieved only if the samples were immersed in water, suggesting that this environment is pivotal for making the surface more compact, possibly by the formation of hydration layers. The contrast may also be enhanced by the presence of chains adsorbed at the tip apex, as suggested by the results in Ref. [17] as well as in recent work by Pawlak et al. [56]. Considering the variety of liquid environments and of physical-chemical conditions which could be addressed in this way (see, e.g., the dynamics of surface reconstructions of metals in electrochemical cells $[57,58]$ ), our results are potentially far reaching and could impact the understanding of friction anisotropy and its relation to the surface microstructure. This could be interesting for the design and fabrication of new and more efficient organic semiconductor devices, such as solar cells, sensors, optically pumped lasers, and stimuli-responsive materials.

\section{ACKNOWLEDGMENTS}

This work was supported by the Spanish Ministry of Economy and Competitiveness (MINECO) (Projects No. MAT2012-38810 and No. CTQ2014-58801), by the Comunidad de Madrid (project Mad2D, Grant No. S2013/MIT3007), by the Campus of International Excellence (CEI) UAM+CSIC, and by the National Research Foundation of Korea (NRF) through a grant funded by the Korean government (MSIP; Grant No. 2009-0081571). The spectroscopic work was performed in the context of the European Cooperation in Science \& Technology Action Nanospectroscopy, MP1302. The authors also acknowledge the support from COST action MP 1303. We thank F. Luo and M. Angel Niño (IMDEA, Madrid) for film preparation by molecular beam epitaxy. S.V. acknowledges funding from the EC via the COFUND program AMAROUT, and C.P. wishes to thank the Spanish government for a FPU Fellowship. M.M. and L.M. acknowledge funding from the French national grant ANR-10-LABX-0042AMADEUS managed by the National Research Agency under the initiative of excellence IdEx Bordeaux program (reference ANR-10-IDEX-0003-02). Computer time for this study was provided by the computing facilities MCIA (Mésocentre de Calcul Intensif Aquitain) of the Université de Bordeaux and of the Université de Pau et des Pays de l'Adour. AFM images taken with Nanoscope Multimode IIIa, Veeco Instruments, were recorded at the ICTS Centro Nacional de Microscopia Electrónica, (UCM) Madrid.
[1] R. P. Steijn, Wear 7, 48 (1964).

[2] F. P. Bowden, C. A. Brookes, and A. E. Hanwell, Nature (London) 203, 27 (1964).

[3] W. G. Sawyer, N. Argibay, D. L. Burris, and B. A. Krick, Annu. Rev. Mater. Res. 44, 395 (2014).

[4] M. Hirano, K. Shinjo, R. Kaneko, and Y. Murata, Phys. Rev. Lett. 67, 2642 (1991).

[5] M. Dienwiebel, G. S. Verhoeven, N. Pradeep, J. W. M. Frenken, J. A. Heimberg, and H. W. Zandbergen, Phys. Rev. Lett. 92, 126101 (2004).

[6] M. Liley, D. Gourdon, D. Stamou, U. Meseth, T. M. Fischer, C. Lautz, H. Stahlberg, H. Vogel, N. A. Burnham, and C. Duschl, Science 280, 273 (1998).

[7] K. Meine, D. Vollhardt, and G. Weidemann, Langmuir 14, 1815 (1998).

[8] K. Hisada and C. M. Knobler, Colloids Surf. A 198, 21 (2002).

[9] S. Freund, A. Hinaut, R. Pawlak, S.-X. Liu, S. Decurtins, E. Meyer, and T. Glatzel, ACS Nano 10, 5782 (2016).

[10] I. Jang, D. L. Burris, P. L. Dickrell, P. R. Barry, C. Santos, S. S. Perry, S. R. Phillpot, S. B. Sinnott, and W. G. Sawyer, J. Appl. Phys. 102, 123509 (2007).

[11] J. S. Choi, J.-S. Kim, I.-S. Byun, D. H. Lee, M. J. Lee, B. H. Park, C. Lee, D. Yoon, H. Cheong, K. H. Lee, Y.-W. Son, J. Y. Park, and M. Salmeron, Science 333, 607 (2011).

[12] P. Gallagher, M. Lee, F. Amet, P. Maksymovych, J. Wang, S. Wang, X. Lu, G. Zhang, K. Watanabe, T. Taniguchi, and D. Goldhaber-Gordon, Nat. Commun. 7, 10745 (2016).
[13] M. Campione, S. Trabattoni, and M. Moret, Tribol. Lett. 45, 219 (2012).

[14] J. Y. Park, D. F. Ogletree, M. Salmeron, R. A. Ribeiro, P. C. Canfield, C. J. Jenks, and P. A. Thiel, Science 309, 1354 (2005).

[15] G. S. Verhoeven, M. Dienwiebel, and J. W. M. Frenken, Phys. Rev. B 70, 165418 (2004).

[16] S. G. Balakrishna, A. S. de Wijn, and R. Bennewitz, Phys. Rev. B 89, 245440 (2014).

[17] Z. Ye, A. Martini, P. Thiel, H. H. Lovelady, K. McLaughlin, and D. A. Rabson, Phys. Rev. B 93, 235438 (2016).

[18] G. Fessler, I. Zimmermann, T. Glatzel, E. Gnecco, P. Steiner, R. Roth, T. D. Keene, S.-X. Liu, S. Decurtins, and E. Meyer, Appl. Phys. Lett. 98, 83119 (2011).

[19] P. Nita, C. Pimentel, F. Luo, B. Milián-Medina, J. Gierschner, C. M. Pina, and E. Gnecco, Nanoscale 6, 8334 (2014).

[20] C. Pimentel, S. Varghese, S.-J. Yoon, S. Y. Park, J. Gierschner, E. Gnecco, and C. M. Pina, J. Phys. Condens. Matter 28, 134002 (2016).

[21] C. M. Pina, R. Miranda, and E. Gnecco, Phys. Rev. B 85, 073402 (2012).

[22] J. G. Vilhena, C. Pimentel, P. Pedraz, F. Luo, P. A. Serena, C. M. Pina, E. Gnecco, and R. Pérez, ACS Nano 10, 4288 (2016).

[23] C. Pimentel, E. Gnecco, and C. M. Pina, Surf. Sci. 635, 123 (2015).

[24] Handbook of Organic Materials for Optical and (Opto)Electronic Devices: Properties and Applications, edited by O. Ostroverkhova (Woodhead, Oxford, 2013). 
[25] Organic Optoelectronics, edited by W. Hu, F. Bai, X. Gong, X. Zhan, F. Hongbing, and T. Bjornholm (Wiley-VCH, Weinheim, Germany, 2013).

[26] Supramolecular Materials for Opto-electronics, edited by N. Koch (RSC Smart Materials, Cambridge, UK, 2015).

[27] R. W. Carpick, D. Y. Sasaki, M. S. Marcus, M. A. Eriksson, and A. R. Burns, J. Phys. Condens. Matter 16, R679 (2004).

[28] P. K.-H. Ho, L.-L. Chua, M. Dipankar, X. Y. Gao, D. C. Qi, A. T.-S. Wee, J.-F. Chang, and R. H. Friend, Adv. Mater. 19, 215 (2007).

[29] M. D. Mowery, S. Kopta, D. F. Ogletree, M. Salmeron, and C. E. Evans, Langmuir 15, 5118 (1999).

[30] V. Kalihari, E. B. Tadmor, G. Haugstad, and C. D. Frisbie, Adv. Mater. 20, 4033 (2008).

[31] V. Kalihari, G. Haugstad, and C. D. Frisbie, Phys. Rev. Lett. 104, 086102 (2010).

[32] J. Y. Park and Y. Qi, Scanning 32, 257 (2010).

[33] K. Voïtchovsky, J. J. Kuna, S. A. Contera, E. Tosatti, and F. Stellacci, Nat. Nanotechnol. 5, 401 (2010).

[34] Z. Ma, Z. Wang, M. Teng, Z. Xu, and X. Jia, Chem. Phys. Chem. 16, 1811 (2015).

[35] K. Ariga, T. Mori, and J. P. Hill, Adv. Mater. 24, 158 (2012).

[36] S.-J. Yoon, J. W. Chung, J. Gierschner, K. S. Kim, M.-G. Choi, D. Kim, and S. Y. Park, J. Am. Chem. Soc. 132, 13675 (2010).

[37] P. Xue, J. Ding, P. Wang, and R. Lu, J. Mater. Chem. C 4, 6688 (2016).

[38] V. Coropceanu, J. Cornil, D. A. da Silva Filho, Y. Olivier, R. Silbey, and J.-L. Brédas, Chem. Rev. 107, 926 (2007).

[39] J. Gierschner, L. Lüer, B. Milián-Medina, D. Oelkrug, and H.-J. Egelhaaf, J. Phys. Chem. Lett. 4, 2686 (2013).

[40] S. Varghese, S.-J. Yoon, S. Casado, R. C. Fischer, R. Wannemacher, S. Y. Park, and J. Gierschner, Adv. Opt. Mat. 2, 542 (2014).

[41] J. Gierschner, S. Varghese, and S. Y. Park, Adv. Opt. Mater. 4, 348 (2015).
[42] I. Horcas, R. Fernández, J. M. Gómez-Rodríguez, J. Colchero, J. Gómez-Herrero, and A. M. Baro, Rev. Sci. Instrum. 78, 13705 (2007).

[43] E. Gnecco and E. Meyer, in Handbook of Nanotechnology, 2nd ed., edited by B. Bhushan (Springer, Berlin, 2007), pp. 923-953.

[44] A. Noy, C. D. Frisbie, L. F. Rozsnyai, M. S. Wrighton, and C. M. Lieber, J. Am. Chem. Soc. 117, 7943 (1995).

[45] J. C. Phillips, R. Braun, W. Wang, J. Gumbart, E. Tajkhorshid, E. Villa, C. Chipot, R. D. Skeel, L. Kalé, and K. Schulten, J. Comput. Chem. 26, 1781 (2005).

[46] J. Wang, R. M. Wolf, J. W. Caldwell, P. A. Kollman, and D. A. Case, J. Comput. Chem. 25, 1157 (2004).

[47] M. Moral, W.-J. Son, J. C. Sancho-García, Y. Olivier, and L. Muccioli, J. Chem. Theory Comput. 11, 3383 (2015).

[48] J. Comer, J. C. Gumbart, J. Hénin, T. Lelièvre, A. Pohorille, and C. Chipot, J. Phys. Chem. B 119, 1129 (2015).

[49] H. Hölscher, A. Schirmeisen, and U. D. Schwarz, Philos. Trans. R. Soc. A 366, 1383 (2008).

[50] M. Campione and E. Fumagalli, Phys. Rev. Lett. 105, 166103 (2010).

[51] M. A. Lantz, S. J. O’Shea, A. C. F. Hoole, and M. E. Welland, Appl. Phys. Lett. 70, 970 (1997).

[52] A. Socoliuc, R. Bennewitz, E. Gnecco, and E. Meyer, Phys. Rev. Lett. 92, 134301 (2004).

[53] J. Gierschner and S. Y. Park, J. Mater. Chem. C 1, 5818 (2013).

[54] E. Gnecco, R. Bennewitz, and E. Meyer, Phys. Rev. Lett. 88, 215501 (2002)

[55] H. Bhaskaran, B. Gotsmann, A. Sebastian, U. Drechsler, M. A. Lantz, M. Despont, P. Jaroenapibal, R. W. Carpick, Y. Chen, and K. Sridharan, Nat. Nanotechnol. 5, 181 (2010).

[56] R. Pawlak, W. Ouyang, A. E. Filippov, L. Kalikhman-Razvozov, S. Kawai, T. Glatzel, E. Gnecco, A. Baratoff, Q. Zheng, O. Hod, M. Urbakh, and E. Meyer, ACS Nano 10, 713 (2015).

[57] A. Labuda, W. Paul, B. Pietrobon, R. B. Lennox, P. H. Grütter, and R. Bennewitz, Rev. Sci. Instrum. 81, 83701 (2010).

[58] F. Hausen, N. N. Gosvami, and R. Bennewitz, Electrochim. Acta 56, 10694 (2011). 